\title{
Strategy for Searching for a Dark Matter Sterile Neutrino
}

\author{
A. Boyarsky, ${ }^{1,2, *}$ A. Neronov, ${ }^{3}$ O. Ruchayskiy, ${ }^{4}$ M. Shaposhnikov, ${ }^{2,1}$ and I. Tkachev ${ }^{1,2}$ \\ ${ }^{1} \mathrm{CERN} / \mathrm{PH}-\mathrm{TH}, \mathrm{CH}-1211$ Geneva 23, Switzerland \\ ${ }^{2}$ École Polytechnique Fédérale de Lausanne, CH-1015, Switzerland \\ ${ }^{3}$ INTEGRAL Science Data Center, Chemin d'Écogia 16, CH-1290 Versoix, Switzerland \\ ${ }^{4}$ Institut des Hautes Études Scientifiques, Bures-sur-Yvette, F-91440, France \\ (Received 3 April 2006; revised manuscript received 20 June 2006; published 27 December 2006)
}

\begin{abstract}
We propose a strategy for how to look for dark matter particles possessing a radiative decay channel and derive constraints on their parameters from observations of $\mathrm{x}$ rays from our own Galaxy and its dwarf satellites. When applied to sterile neutrinos in the $\mathrm{keV}$ mass range this approach gives a significant improvement to restrictions on neutrino parameters compared with previous works.
\end{abstract}

DOI: 10.1103/PhysRevLett.97.261302

Introduction. - It was noticed long ago that a sterile neutrino with mass in the $\mathrm{keV}$ range appears to be a viable dark matter (DM) candidate [1]. Moreover, being warm $\mathrm{DM}$, a sterile neutrino eases the tension between observations and predictions of the cold DM model on small scales. The interest in this scenario has been revived since the discovery of neutrino oscillations (see, e.g., [2] for a review). Indeed, one of the simplest ways to explain these data is to add to the standard model (SM) several gauge singlet fermions - right-handed, or sterile, neutrinos. It has been demonstrated recently [3] that a simple extension of the SM by three singlet fermions with masses smaller than the electroweak scale, dubbed the $\nu$ MSM in [3], allows one to describe all confirmed data on neutrino oscillations, provides a DM particle candidate in the form of a sterile neutrino, and allows one to explain the baryon asymmetry of the Universe. The simplicity of the model, the similarity of its quark and lepton right-handed sectors, together with a considerable number of other phenomena it can simultaneously describe, forces us to take this model seriously and thus provides additional motivation for the study of $\mathrm{keV}$ mass range sterile neutrinos as a DM candidate.

The sterile neutrino has a radiative decay channel, emitting a photon with energy $E=m_{s} / 2\left(m_{s}\right.$ being the mass of the sterile neutrino). Parametrically, the decay width is proportional to $m_{s}^{5} \sin ^{2} 2 \theta$ [4], where $\theta$ is the mixing angle between active and sterile neutrino.

If such a neutrino is a main ingredient of the DM, it is potentially detectable in various $x$-ray observations. The most obvious candidates are diffuse extragalactic $\mathrm{x}$-ray background (XRB) [5-8], clusters of galaxies $[6,9,10]$, and galaxies [6], including our own.

The aim of the present Letter is to discuss the best strategy to search for a DM sterile neutrino and to derive the constraints on its properties. Although we concentrate on the sterile neutrino, the constraints we get can be applied to any DM candidate with a radiative two-body decay channel in the $\mathrm{keV}$ range. We analyze various types of astrophysical objects and show that the strongest constraints on sterile neutrino come from neutrino decays in the Milky Way halo and, in particular, in the halo dwarf
PACS numbers: 95.35.+d, 14.60.Pq, 95.85.Nv

galaxies. These objects were not considered previously in this context. The existing XMM-Newton and HEAO-1 data allow us to improve over previous constraints, highlighting the potential of new optimal sites for the searches of the signal from the sterile neutrino decay.

Dark matter halo of the Milky Way. - The energy flux produced by the DM decay from a given direction into a solid angle $\Omega_{\text {fov }} \ll 1$ is given by

$$
F=\frac{\Gamma \Omega_{\text {fov }}}{8 \pi} \int_{\text {line of sight }} \rho_{\mathrm{DM}}(r) d r,
$$

where $\Gamma$ is the radiative decay width of the sterile neutrino. To determine the Milky Way (MW) contribution into the flux (1), one needs to know the distribution of the DM in the halo.

Mass distribution within MW has been modeled by many authors. Characteristics which are relevant for our study are tightly constrained by the wealth of detailed data available for this system. As a reference we will choose the mass distribution derived recently in [11], where physically interesting models were selected by imposing additional constraints based on a theory of halo formation. At large $r$ the halo density can be described by the NavarroFrenk-White (NFW) profile $\rho_{\mathrm{NFW}}(r)=\frac{M_{\mathrm{vir}}}{4 \pi \alpha} \frac{1}{r\left(r_{s}+r\right)^{2}}$. The MW halo parameters of favorite models obtained in [11] correspond to $M_{\mathrm{vir}}=1.0 \times 10^{12} M_{\odot}, r_{s}=21.5 \mathrm{kpc}$ and the numerical constant $\alpha \simeq 1.64$.

In the region of $r$ relevant for our study the halo density can be also approximated by the isothermal profile

$$
\rho_{\text {halo }}=\frac{v_{h}^{2}}{4 \pi G_{N}} \frac{1}{r_{c}^{2}+r^{2}},
$$

where $v_{h}$ corresponds to the contribution of the DM halo into the Galactic rotation curve in its flat part, $v_{h} \approx$ $170 \mathrm{~km} / \mathrm{s}$; see, e.g., [11].

The NFW density profile and (2) produce identical fluxes from the direction of the Galactic anticenter if $r_{c} \approx$ $4 \mathrm{kpc}$, giving $\int_{r_{\odot}}^{\infty} \rho_{\text {halo }} d r \approx 0.7 \times 10^{22} \mathrm{GeV} / \mathrm{cm}^{2}$. They also closely follow each other in the range $3 \mathrm{kpc}<r<$ $80 \mathrm{kpc}$ (the difference being less than 5\%). Therefore, in 
estimates of the flux from directions that are outside of a $20^{\circ}$ circle around the Galactic center both NFW and (2) give the same results.

The halo density profile is less certain in the region $r<$ $r_{\odot}$. In [11] two distinct types of models were considered, with and without exchange of angular momentum between DM and baryons. In the model without momentum exchange, the DM density profile at $r<10 \mathrm{kpc}$ diverges even faster than NFW profile. In the model with angular momentum exchange the DM density profile at $2 \mathrm{kpc}<r<$ $10 \mathrm{kpc}$ is less singular and rather resembles the isothermal sphere Eq. (2) with $r_{c} \approx 4 \mathrm{kpc}$, but the DM density is larger at $r<2 \mathrm{kpc}$ as compared to the isothermal sphere. Therefore, one can use Eq. (2) with $r_{c}=4 \mathrm{kpc}$ as a lower limit on the DM density when calculating fluxes from DM decays, and, therefore, for putting a conservative bound on the sterile neutrino mixing angle [e.g., the halo density at the Sun position in the model Eq. (2) is $0.25 \mathrm{GeV} / \mathrm{cm}^{3}$, which is smaller than the accepted value $0.3 \mathrm{GeV} / \mathrm{cm}^{3}$ [12] ]. Using the NFW profile at all $r$ can only strengthen the bounds.

In the model Eq. (2) the DM flux from the direction $(b, l)$ (in galactic coordinates) into the solid angle $\Omega_{\text {fov }} \ll 1$, measured by an observer on Earth is given by

$$
F_{\mathrm{MW}}(\phi)=\frac{L_{0}}{R} \times \begin{cases}\frac{\pi}{2}+\arctan \left(\frac{r_{\odot} \cos \phi}{R}\right), & \phi \in\left[0: \frac{\pi}{2}\right] \\ \arctan \left(\frac{R}{\left.r_{\odot} \mid \cos \phi\right)}\right), & \phi \in\left[\frac{\pi}{2}: \pi\right],\end{cases}
$$

where $L_{0} \equiv \frac{\Gamma \Omega_{\mathrm{fov}} v_{h}^{2}}{32 \pi^{2} G_{N}}, R=\sqrt{r_{c}^{2}+r_{\odot}^{2} \sin ^{2} \phi}$, and $\cos \phi=$ $\cos b \cos l$. For example, $F_{\mathrm{MW}}\left(90^{\circ}\right) / F_{\mathrm{MW}}\left(180^{\circ}\right) \simeq 1.52$.

Search for a preferred observation. - Let us compare the Galaxy contribution to the DM decay flux computed above, with that of other astrophysical objects.

(i) XRB. - Although the DM has a very narrow radiative decay width, the cosmological DM decay contribution to the XRB broadens due to the contributions from various redshifts. The paper [5] looked at restrictions from XRB, assuming that DM is uniform up to very small $z$. This question was further addressed in [6,7] and finally the most stringent constraint in the $\left(\sin ^{2} 2 \theta, m_{s}\right)$ plane from XRB was obtained recently in [8].

One can compare the expected contribution of the DM decay to the total XRB flux with the galactic contribution: $F_{\mathrm{MW}} / F_{\mathrm{XRB}}=\mathcal{R} r_{c} H_{0}$, where $\mathcal{R} \sim \rho_{\mathrm{MW}}^{0} / \rho_{\mathrm{DM}}^{0} \sim 10^{6}$ is the overdensity of the Galaxy as compared to the average density of DM in the Universe. With $r_{c} \sim 4 \mathrm{kpc}$, one arrives to the conclusion that $F_{\mathrm{MW}} / F_{\mathrm{XRB}} \sim 1$; i.e., the galactic contribution is comparable with the total DM decay flux from all redshifts.

However, for a modern $\mathrm{x}$-ray instrument with good spectral resolution $\Delta E \ll m_{s}$ (e.g., XMM-Newton) one should compare contributions from the Galaxy and from a uniform cosmological distribution into XRB within $\Delta E$. The ratio $F_{\mathrm{MW}} / F_{\mathrm{XRB}}$ then gets enhanced by the factor $E / \Delta E$ which is $10-50$ for EPIC cameras on board of $\mathrm{XMM}-N$ ewton; i.e., for XMM the Galactic signal is 1 to
2 orders of magnitude stronger than the contribution from the uniform distribution of DM in the Universe.

(ii) Clusters. - Let us now analyze the flux enhancement from Coma and Virgo clusters of galaxies [6,10]. Using, e.g., results of [10], we get $F_{\mathrm{MW}}\left(90^{\circ}\right) / F_{\mathrm{Coma}} \approx 0.25$. Similar estimate for the center of the Virgo cluster shows that Galactic contribution is $\sim 10 \%$. Therefore, one could conclude that clusters are preferable objects for DM detection $[6,9]$.

These results have been reanalyzed in [10], where it was shown that the actual improvement from Coma and Virgo clusters over XRB is on average factor $\sim 7\left[\sin ^{2}(2 \theta)\right.$ for given $m_{s}$ ]. However, the search for the DM decay line in clusters is complicated by the fact that the clusters of galaxies are x-ray bright objects. Indeed, the virial theorem shows that the temperature of the intercluster medium is $T_{\text {gas }} \sim G_{N} m_{p} \mathcal{R} \rho_{\mathrm{DM}}^{0} d^{2}$, where $d$ is the characteristic size. For overdensity $\mathcal{R} \sim 10^{3}$ and size $d \sim 1 \mathrm{Mpc}$, the temperature $T_{\text {gas }}$ falls into the $\mathrm{keV}$ range, which makes it hard to detect a DM decay line against a strong x-ray continuum.

(iii) Dwarf galaxies. - There should be an enhancement of the flux in directions of dwarf spheroidal galaxies, which are satellites of the MW. Promising satellites with large mass to light ratio are Draco and Ursa Minor. Density profiles of both galaxies can be modeled by the isothermal sphere with $v_{h} \approx 22 \mathrm{~km} / \mathrm{s}$ and $r_{c} \approx 100 \mathrm{pc}$ [13]. This gives for the contribution to the flux from the dwarf galaxy (along the line which passes through the core of the satellite) $\int \rho d r \approx 3.3 \times 10^{22} \mathrm{GeV} / \mathrm{cm}^{2}$. One can see, for example (c.f. [14]), that adopted numbers provide a lower bound on DM flux among currently admissible DM models for Draco dwarf.
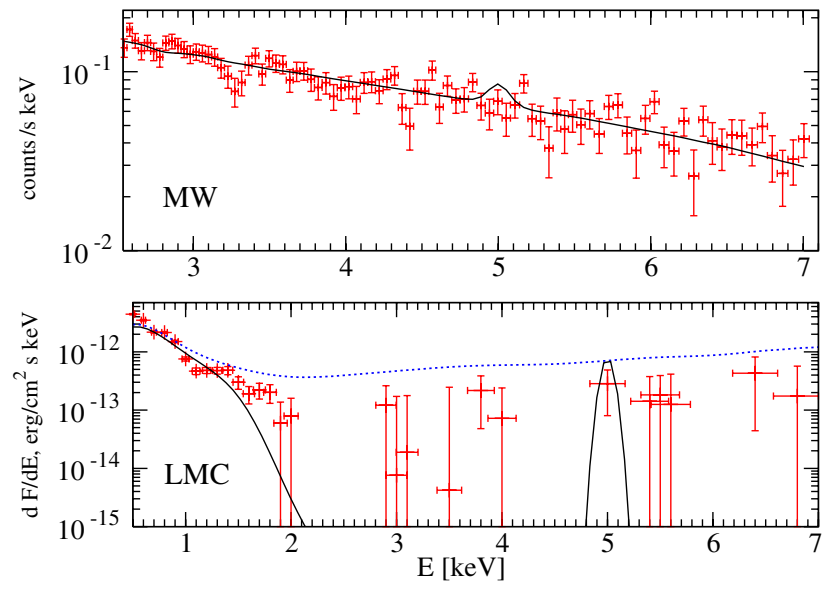

FIG. 1 (color online). Upper panel: Method used to obtain restrictions on DM decay in MW from blank sky XRB observations. The data are fitted by a power law (reduced $\chi^{2}=1.07$ for 82 d.o.f.) and XSPEC v11.3.2 is used to put a $3 \sigma$ limit on the presence of DM line (via command "error <line norm> 9,0"). Lower panel: Method used to obtain restrictions from LMC. Flux rapidly decreases for $E \gtrsim 2 \mathrm{keV}$, most of the data points at higher energies are zero within statistical uncertainty. The dotted line is the sum of the total flux plus $3 \sigma$ per energy bin. 


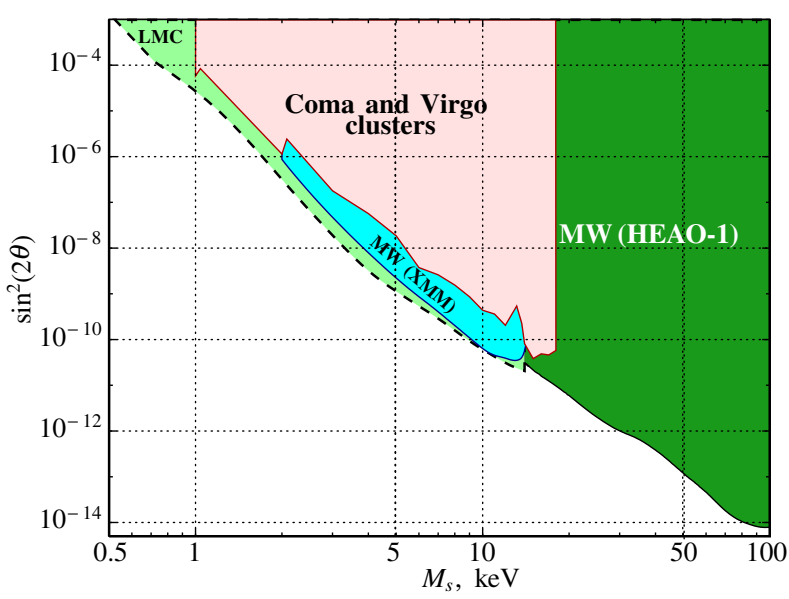

FIG. 2 (color online). $3 \sigma$ restrictions on DM decay line from Milky way halo (XMM and HEAO-1 observations). Dashed line: total flux $+3 \sigma$ restriction from LMC. Also shown the previous strongest limit from the clusters of galaxies [10].

Contribution of the Galaxy halo flux in the directions of both satellites $\approx 1.0 \times 10^{22} \mathrm{GeV} / \mathrm{cm}^{2}$. Therefore, in directions of both satellites the fourfold local enhancement of the flux is expected, while the total flux matches the flux from the central region of the MW and the flux from clusters. The advantage of observing the dwarf satellites, as compared to clusters or to the Galactic center, is a lower level of x-ray background contamination and a clear signature of the signal, namely, local flux enhancement within a single field of view of an $\mathrm{x}$-ray telescope.

Therefore, we see that the local halo (especially dwarf satellite galaxies) can provide the strongest restriction on the parameters of the sterile neutrino as DM candidates.

Restrictions from local halo DM contribution.-We have analyzed the XMM blank sky observations of [15] (exposure time $\sim 200 \mathrm{ksec}$ ), taking into account the MW contribution and putting a $3 \sigma$ bound on the DM line flux (Fig. 1). We chose to work in the region $1 \mathrm{keV}<E<$ $7 \mathrm{keV}$, where uncertainties of the instrumental background normalization (c.f. [16]) lead to the error on derived parameters $\leqslant 30 \%$. The exclusion plot in the region $m_{s}>$ $6 \mathrm{keV}$ was obtained by using the HEAO- 1 measurements of XRB [17]. As spectral resolution of HEAO-1 is about $25 \%$, the resulting correction due to MW is not so drastic as compared to the results of Ref. [8].

Unfortunately, x-ray observations of Draco and Ursa Minor dwarfs are not available currently. However, approximately the same signal is expected from the Large Magellanic Clouds (LMC) albeit with larger uncertainties. Using isothermal sphere with the halo parameters $v_{h} \approx$ $50 \mathrm{~km} / \mathrm{s}$ and $r_{c} \approx 1 \mathrm{kpc}$ [18], we obtain $\int \rho d r \approx 2.8 \times$ $10^{22} \mathrm{GeV} / \mathrm{cm}^{2}$ for the LMC contribution, while MW halo contribution in the direction of LMC is again $\approx 1.0 \times$ $10^{22} \mathrm{GeV} / \mathrm{cm}^{2}$ (the use of the NFW profile gives even larger value for flux).

Therefore, as an illustration we have processed one of the observations of the LMC (XMM obs ID: 0127720201, exposure $\sim 20 \mathrm{ksec}$ ). Subtracting the blank sky background [15], one sees that the flux is zero within statistical uncertainty for $E \gtrsim 2 \mathrm{keV}$. A similar reduction of the background is expected for dwarf satellites. In this situation we put an upper limit on the flux of the DM decay line, by demanding it to be smaller than total flux plus its $3 \sigma$ error in an energy bin equal to spectral resolution; see Fig. 1. Increasing the exposure time, one can significantly lower the restriction in the region $E \geqslant 2 \mathrm{keV}$. An additional source of error in this procedure is the uncertainty of the blank sky background normalization [16]. In our case this error is smaller than the statistical error in the measured LMC flux.

Discussion. - In this Letter we have shown that the best objects for the search of the DM with radiative decay channel is the MW halo, including dwarf satellite galaxies (e.g., Draco and Ursa Minor). Moreover, we have found, quite remarkably, that the line of sight integral Eq. (1) is roughly the same for all studied DM dominated objects, from cosmological background to clusters of galaxies to dwarfs satellites. This suggests that the expected DM decay signal should be roughly the same for all of them. Better constraints are obtained for those objects whose $\mathrm{x}$-ray background is lower. This makes Milky Way and its dwarf satellites more suitable as compared to clusters.

To illustrate this, we put restrictions on parameters of the sterile neutrino (i) searching for the MW DM decay signal in the blank sky XRB, and (ii) using LMC observations; see Fig. 2. One can see that improving statistics one can significantly strengthen bounds from such objects. Our analysis also shows that improvement of the spectral resolution of x-ray instruments (even by means of decreasing imaging capabilities) is crucial to continuing the search of DM decay line.

Of course, constraints on the sterile neutrino parameters, derived from x-ray observations, suffer from uncertainties in the DM profiles. Therefore, we tried to be conservative in determining the DM contribution. For example, in the case of LMC the NFW DM profile from [19] would give $\sim 4$ times stronger restrictions on $\sin ^{2}(2 \theta)$ than those presented here. However, the modeling of LMC is subject to debate in the literature. It should be stressed that our result can be relaxed if the actual DM fraction of LMC is smaller than we assumed. In the case of MW, the expected DM signal from the Galactic center is 6 times stronger than that from the anticenter. However, the fraction (and distribution) of DM in the direction of the Galactic center are much more model dependent and uncertain. Different profiles discussed in [11] and in the more recent work [20] give consistent (within 5\%) values of DM flux in the anticenter direction, used in the present work. If future studies determine different parameters of DM distributions in these objects, our limits will be rescaled correspondingly.

As the existence of the DM is deduced from the analysis of many objects of different types and scales, the bounds on the DM parameters should also be derived from studying not one, but many objects and, moreover, various 
classes of objects. For example, when the data become available, it will be important to derive a bound not from LMC only, but from several dwarf galaxies. This will make the uncertainties in the DM modeling in each individual object less important. In addition, in the most DM dominated satellites, such as Draco and Ursa Minor, the uncertainties due to subtraction of baryonic component from the galactic rotational curve are also minimized. However, following the same logic as above, we would like to stress again that independent bounds from different types of objects are important, even if some of them give weaker restrictions.

The limits we derived here allow to us strengthen the bounds on the sterile neutrinos in different models of particle physics, shed light on the possible mechanisms of their production in the early Universe, and constrain different astrophysical phenomena that might be related to their existence.

(i) Assuming the absence of the sterile neutrinos above the temperature $\sim 1 \mathrm{GeV}$ and charge neutrality of the plasma, the relic abundance of sterile neutrinos can be expressed through $m_{s}$ and $\theta$ in the SM with just one sterile neutrino added [1] and in the $\nu$ MSM [21]. This relation (quite uncertain, since the sterile neutrinos are mainly produced at temperatures $\mathcal{O}(150) \mathrm{MeV}$, where the description of the strongly interacting plasma is most complicated [22]) allows one to find, potentially, an upper limit on the sterile neutrino mass in this particular scenario. Taking as a rough estimate the computation of [9] and our x-ray constraints we arrive to an upper bound $m_{s} \lesssim 3 \mathrm{keV}$. This may be contrasted with the lower bound on the mass of the sterile neutrino (derived in the same model with the same assumptions) coming from the analysis of the Ly- $\alpha$ forest data [23]: $m_{s}>2 \mathrm{keV}$ [24], $m_{s}>1.7 \mathrm{keV}$ [25] leaving a very limited allowed mass range for the sterile neutrino. If a more recent result of [26], $m_{s}>14 \mathrm{keV}$ is proven to be correct, and uncertainties related to poor knowledge of QCD happen to be not substantial, this scenario will be ruled out by cosmological and astrophysical observations. This would make the production mechanisms of the sterile neutrinos related to inflation [27] or large lepton asymmetries [28] more important. (ii) In [29] it was shown that the XRB limits of [8] imply that the lightest active neutrino must have a mass $m_{\nu}<3 \times 10^{-3} \mathrm{eV}$, provided $m_{s}>$ $1.8 \mathrm{keV}$. In the case of normal hierarchy the two other masses are given by the observed mass square differences $\sqrt{\Delta m_{\text {solar }}^{2}}$ and $\sqrt{\Delta m_{\text {atm }}^{2}}$ (in the case of inverted hierarchy both neutrinos have mass $\sqrt{\Delta m_{\mathrm{atm}}^{2}}$ ). With the improved bound, derived in this Letter, this result is true for sterile neutrino masses $m_{s}>1.3 \mathrm{keV}$. (iii) Our constraints, combined with those of [26], put severe bounds on the explanation of pulsar kick velocities and on the mechanism of early reionization by sterile neutrinos (for discussion see [30] and references therein).

We thank A. Kusenko and M. Markevitch for discussions. M.S. was supported in part by the Swiss Science Foundation.
Note added. -After finishing this Letter we learned about work [31], which studies DM decay signal from MW using Chandra blank sky data.

*On leave from Bogolyubov Institute of Theoretical Physics, Kyiv, Ukraine.

[1] S. Dodelson and L. M. Widrow, Phys. Rev. Lett. 72, 17 (1994).

[2] A. Strumia and F. Vissani, hep-ph/0606054.

[3] T. Asaka, S. Blanchet, and M. Shaposhnikov, Phys. Lett. B 631, 151 (2005); T. Asaka and M. Shaposhnikov, ibid. 620, 17 (2005).

[4] P. B. Pal and L. Wolfenstein, Phys. Rev. D 25, 766 (1982); V. D. Barger, R. J. N. Phillips, and S. Sarkar, Phys. Lett. B 352, 365 (1995).

[5] A. D. Dolgov and S. H. Hansen, Astropart. Phys. 16, 339 (2002).

[6] K. Abazajian, G. M. Fuller, and W. H. Tucker, Astrophys. J. 562, 593 (2001).

[7] M. Mapelli and A. Ferrara, Mon. Not. R. Astron. Soc. 364, 2 (2005).

[8] A. Boyarsky et al., Mon. Not. R. Astron. Soc. 370, 213 (2006).

[9] K. Abazajian, Phys. Rev. D 73, 063506 (2006).

[10] A. Boyarsky et al., Phys. Rev. D 74, 103506 (2006).

[11] A. Klypin, H. Zhao, and R. S. Somerville, Astrophys. J. 573, 597 (2002).

[12] S. Eidelman et al., Phys. Lett. B 592, 1 (2004).

[13] M. I. Wilkinson et al., astro-ph/0602186.

[14] S. Mashchenko, A. Sills, and H. M.P. Couchman, Astrophys. J. 640, 252 (2006).

[15] A. M. Read and T. J. Ponman, Astron. Astrophys. 409, 395 (2003).

[16] J. Nevalainen, M. Markevitch, and D. Lumb, Astrophys. J. 629, 172 (2005).

[17] D. E. Gruber et al., Astrophys. J. 520, 124 (1999).

[18] R. van der Marel et al., Astrophys. J. 124, 2639 (2002).

[19] A. Tasitsiomi, J. Gaskins, and A. V. Olinto, Astropart. Phys. 21, 637 (2004).

[20] G. Battaglia et al., Mon. Not. R. Astron. Soc. 364, 433 (2005) 370, 1055(E) (2006).

[21] T. Asaka, M. Shaposhnikov, and A. Kusenko, Phys. Lett. B 638, 401 (2006).

[22] T. Asaka, M. Laine, and M. Shaposhnikov, J. High Energy Phys. 06 (2006) 053.

[23] S. H. Hansen et al., Mon. Not. R. Astron. Soc. 333, 544 (2002).

[24] M. Viel et al., Phys. Rev. D 71, 063534 (2005).

[25] K. Abazajian, Phys. Rev. D 73, 063513 (2006).

[26] U. Seljak et al., Phys. Rev. Lett. 97, 191303 (2006).

[27] M. Shaposhnikov and I. Tkachev, Phys. Lett. B 639, 414 (2006).

[28] X.d. Shi and G. M. Fuller, Phys. Rev. Lett. 82, 2832 (1999).

[29] A. Boyarsky et al., JETP Lett. 83, 133 (2006).

[30] A. Kusenko, Phys. Rev. Lett. 97, 241301 (2006).

[31] S. Riemer-Sorense, S.H. Hansen, and K. Pedersen, Astrophys. J. 644, L33 (2006). 